\title{
Atrial fibrillation and COVID-19 in older patients: how disability contributes to shape the risk profile. An analysis of the GeroCovid registry
}

\author{
Stefano Fumagalli ${ }^{1} \mathbb{D}$. Caterina Trevisan ${ }^{2}$. Susanna Del Signore ${ }^{3}$. Giulia Pelagalli ${ }^{1}$. Carlo Fumagalli ${ }^{1}$. \\ Andrea Herbst ${ }^{1}$ - Stefano Volpato ${ }^{4}$. Pietro Gareri ${ }^{5}$. Enrico Mossello ${ }^{1}$ - Alba Malara ${ }^{6}$. Fabio Monzani ${ }^{7}$. \\ Chukwuma Okoye ${ }^{7}$. Alessandra Coin ${ }^{2}$. Giuseppe Bellelli ${ }^{8}$. Gianluca Zia ${ }^{3}$. Andrea Ungar ${ }^{1}$. Anette Hylen Ranhoff ${ }^{9}$. \\ Raffaele Antonelli Incalzi ${ }^{10} \cdot$ for the GeroCovid Working Group
}

Received: 22 August 2021 / Accepted: 16 October 2021 / Published online: 30 October 2021

(c) The Author(s), under exclusive licence to Springer Nature Switzerland AG 2021

\begin{abstract}
Background and aims Atrial fibrillation (AF) is often complicated by disabling conditions in the elderly. COVID-19 has high mortality in older people. This study aimed at evaluating the relationship of pre-infection AF with characteristics and survival of older COVID-19 patients.

Methods We retrospectively analyzed inpatients aged $\geq 60$ years enrolled in GeroCovid Observational, a multicenter registry endorsed by the Italian and the Norwegian Societies of Gerontology and Geriatrics. Pre-COVID-19 sociodemographic, functional, and medical data were systematically collected, as well as in-hospital mortality.

Results Between March and June 2020, 808 COVID-19 subjects were enrolled (age $79 \pm 9$ years; men 51.7\%). The prevalence of $\mathrm{AF}$ was $21.8 \%$. AF patients were older ( $82 \pm 8$ vs. $77 \pm 9$ years, $p<0.001)$, had a higher $\mathrm{CHA}_{2} \mathrm{DS}_{2}-\mathrm{VASc}$ score $(4.1 \pm 1.5$ vs. $3.2 \pm 1.5, p<0.001)$ and were more likely to present almost all comorbidities. At multivariable analysis, advanced age, white blood cell count, the presence of heart and peripheral artery diseases were significantly associated with the presence of AF. In-hospital mortality was higher in AF patients (36.9 vs. 27.5\%; $\mathrm{OR}=1.55,95 \% \mathrm{CI}=1.09-2.20 ; p=0.015)$. A decision tree analysis showed that, in AF subjects, preserved functional status at admission was the most important factor associated with survival. In patients without AF, baseline COVID-19 severity was the most relevant variable related to clinical prognosis. Conclusions AF is frequent in older patients with COVID-19, in whom it associates with clinical complexity and high mortality. Pre-infection disability shapes the prognosis of this extremely vulnerable segment of hospitalized subjects.

Clinical trial registration GeroCovid Observational was registered at www.clinicaltrials.gov (NCT04379440).
\end{abstract}

Keywords Atrial fibrillation $\cdot$ COVID-19 $\cdot$ Disability $\cdot$ Older patients $\cdot$ Oral anticoagulants $\cdot$ Prognosis

Stefano Fumagalli

stefano.fumagalli@unifi.it

1 Geriatric Intensive Care Unit and Geriatric Arrhythmia Unit, Department of Experimental and Clinical Medicine, University of Florence and AOU Careggi, Largo Brambilla, 3, 50134 Florence, Italy

2 Geriatrics Division, Department of Medicine (DIMED), University of Padua, Padua, Italy

3 Bluecompanion Ltd, London, UK

4 Department of Medical Science, Section of Internal and Cardiorespiratory Medicine, University of Ferrara, Ferrara, Italy

5 Center for Cognitive Disorders and Dementia-Catanzaro Lido, ASP Catanzaro, Catanzaro, Italy
6 Scientific Committee of National Association of Third Age Residences (ANASTE) Calabria, Lamezia Terme (Catanzaro), Italy

7 Geriatrics Unit, Department of Clinical and Experimental Medicine, University of Pisa, Pisa, Italy

8 School of Medicine and Surgery, Acute Geriatric Unit, University of Milano-Bicocca, San Gerardo Hospital, Monza, Italy

9 Department of Clinical Science, University of Bergen, Bergen, Norway

10 Unit of Geriatrics, Department of Medicine, Campus Bio Medico University and Teaching Hospital, Rome, Italy 


\section{Introduction}

Atrial fibrillation (AF) is the most frequent sustained arrhythmia observed in clinical practice. Its incidence and prevalence significantly increase with age. Several population studies demonstrated that the presence of AF was associated with reduced survival, especially in older individuals [1]. The coronavirus disease 2019 (COVID-19) pandemic correlated with a dramatic upsurge of mortality, which was particularly evident in the oldest segments of population, those most commonly affected by AF and related comorbidities. Indeed, age per se resulted as one of the most powerful risk factors of unfavorable in-hospital prognosis in almost all studies [2]. Furthermore, some evidence exists linking AF and COVID-19, showing that after the pandemic outbreak the incidence of the arrhythmia was 33\% higher than the year before [3]. These results could be explained by the prolonged time spent at home, resulting in physical inactivity, a higher rate of obesity and the rise of depressive symptoms [3]. The analysis of large prospective medical datasets further confirmed the association between COVID19 and incident AF [4]. Age and a previous arrhythmic event were significant predictors of AF development in patients hospitalized for the infection [5]. A meta-analysis and two studies evaluating patients younger than ours found that both pre-existing and incident AF were related to increased COVID-19 mortality [6-8]. In a polycentric Italian experience, including a population $\geq 18$ years, the arrhythmia (in clinical history or new-onset) was associated with all-cause mortality in hospitalized patients, independently of older age [9]. Lastly, in a previous analysis of the GeroCovid registry, we observed that the use of oral anticoagulation prior to the hospitalization for SARS-CoV-2 infection positively correlated with survival in aged AF individuals [10], evidence not found in a younger population [8]. On this basis, we planned a comprehensive assessment of the clinical characteristics and risk profile of older COVID-19 patients with and without $\mathrm{AF}$ in order to define whether and, possibly, through which mechanisms, AF and age could interact affecting inhospital survival. Detecting distinctive associations linking COVID-19, AF and prognosis could help to more appropriately manage this complex and frail segment of population affected by the pandemic.

\section{Methods}

\section{Study population}

As previously described [11], GeroCovid Observational (ClinicalTrials.gov registration: NCT04379440) is a multi-purpose and multicenter study registry endorsed by the Italian Society of Gerontology and Geriatrics (SIGG) and the Norwegian Geriatrics Society aiming at investigating the impact of SARS-CoV-2 infection in older patients in different settings of care. The final endpoint of GeroCovid Observational is to provide high-quality and comprehensive data, to help and optimize COVID-19 prevention and management of patients $\geq 60$ years. Among the secondary outcomes of the project, special attention is given to study the effects of the interaction between virus infection and multi-morbidity on mortality and other negative health-related endpoints. Overall, 66 investigational sites are actively participating. By protocol, data can be collected retrospectively and prospectively in an e-Registry since March 1st, 2020. The objectives of GeroCovid are specific for each setting of care [11]. The participation of each center was approved by the corresponding local Ethical Committee.

For the present analysis, we included, almost always retrospectively (96.3\% of the whole population), patients hospitalized in the 16 centers participating at the "GeroCovid acute wards" cohort of the registry during the first wave of the COVID-19 pandemic [11]. Informed consent was obtained from all participants included in the study or from their legal caregivers. Because of the privacy of individuals that were enrolled, the particular severe clinical conditions we observed in many cases, and the on-going follow-up, the data underlying this article cannot be shared publicly. The data will be shared on reasonable request to the corresponding author.

\section{Data collection}

For each participant, the presence of AF was derived either from the medical history or from hospital records at ward admission. Complete information on chronic diseases and pre-admission pharmacological therapy was obtained from medical records. Similarly, data on COVID-19 symptoms/ signs at the disease onset, vital signs (blood pressure, heart rate) and biochemical analyses were collected at baseline. To assess pre-COVID-19 functional status, a 7-level scale was used. For the purpose of the study, given the distribution of the variable in our population, we categorized patients as without vs. with functional limitations, by grouping levels 1 and 2 (the patient can walk independently or with a cane) and levels 3-7 (the patient can walk using a walker; the patient can move around with a wheelchair; the patient does not move around but he is accompanied outside on the wheelchair; the patient is confined at home, mostly lying on the bed; the patient does not autonomously stand up or get in sitting position) [10]. According to the World Health Organization (WHO) classification and on patients' distribution, the baseline severity of COVID-19 was defined as 
mild with no oxygen support needed, mild with low-flow oxygen support needed, and severe or critical (high-flow oxygen support required, need of non-invasive or invasive mechanical ventilation, or organ support) [10]. Finally, data on in-hospital mortality were also recorded for all the study participants.

\section{Statistical analysis}

IBM SPSS ver. 27.0 (64-bit edition) for macOS was used for statistical analysis. Continuous variables are expressed as mean $\pm \mathrm{SD}$, while discrete variables as raw numbers and percentages. The comparison of continuous variables in patients with and without AF was performed with the Student's t-test or the Mann-Whitney test, as appropriate. General Linear Univariate Models were used to test the association of AF, in-hospital mortality and their interaction with length of stay in hospital. For categorical variables, we used the Chisquare test.

We built a multivariable logistic regression analysis model to identify the factors independently associated with the presence of AF. The least statistically correlated variables were iteratively removed, using a backward deletion process. Those factors showing high collinearity with others, but a weaker association with arrhythmia were also excluded. The strength of the associations between the factors identified as significant and $\mathrm{AF}$ was expressed as odds ratios (ORs) and 95\% confidence intervals (95\% CIs).

To study the influence of AF on mortality through a multivariable approach, we used a tree-based classification model, with in-hospital survival as the dependent variable, and forcing the presence of AF as the first stratifying independent variable. Age, gender, obesity, functional dependency before hospital admission, residence in nursing homes, $\mathrm{CHA}_{2} \mathrm{DS}_{2}$-VASc score, history of chronic obstructive pulmonary disease (COPD) and chronic kidney disease (CKD), baseline COVID-19 status according to WHO classification, heart rate and systolic arterial pressure at admission, and, last, pre-infection use of oral anticoagulant therapy and renin-angiotensin system antagonists were included in the model. At each step of the analysis, the Chi-squared Automatic Interaction Detection (CHAID) growing method identified the independent variables that had the strongest interaction with in-hospital prognosis. Accordingly, this process permitted to progressively divide and classify the initial population into different groups, characterized by the highest association with mortality. On the basis of the results that were obtained, GeroCovid population was then ranked into separate clusters characterized by different survival rates. This allowed to quantify models' quality using the Area Under the ROC Curve (AUC). For all analyses, statistical significance was set at a $p$-value $<0.05$.

\section{Results}

From March 1st to June 6th 2020, 2474 patients were enrolled in the GeroCovid Registry; $808(N=32.6 \%)$ of these were hospitalized in an acute care setting and were analyzed in the present study.

Atrial fibrillation (AF) at admission or in clinical history was present in 176 cases $(21.8 \%)$.

\section{Clinical characteristics by AF status}

Compared with non-AF patients, those with the arrhythmia were older and had a worse pre-COVID-19 functional profile. Overall, no differences were observed in body size by rhythm, even if frank malnutrition and obesity were more common with AF (Table 1 and Fig. 1).

Comorbidities were more frequently observed in AF patients (Fig. 1). In particular, both cardiovascular (i.e., the presence of any cardiac disorder, signs and symptoms of chronic heart failure, peripheral artery disease and stroke) and non-cardiovascular conditions (i.e., arthritis, CKD, COPD, diabetes) had a higher prevalence in AF patients. The $\mathrm{CHA}_{2} \mathrm{DS}_{2}$-VASc score was also higher in those with the arrhythmia (Table 1).

Anti-arrhythmic agents, beta-blockers, digitalis, diuretics and oral anticoagulants were more often prescribed in patients with AF. The proportion of AF subjects who were anticoagulated was $51.7 \%$, with DOACs preferred on VKAs. The use of anti-platelet agents was slightly higher in non-AF patients, while no differences were reported in the use of antagonist of the renin-angiotensin system and in the severity of COVID-19 according to WHO classification (Table 1). Among blood tests, $\mathrm{Hb}$ concentration was greater in non-AF, and white blood cell (WBC) count was higher in AF group, while lactate dehydrogenase and C-reactive protein levels did not differ by rhythm.

Concerning COVID-19 signs or symptoms, cough, fever and hemoglobin oxygen desaturation $(<90 \%$ in ambient air) when walking were more common in non-AF patients (Table 2). A lower possibility to talk was more frequently observed in AF subjects (21.0 vs. $12.8 \% ; p=0.023$ ).

At multivariable logistic regression analysis (overall predictivity: $82.9 \%, p<0.001$ ), the presence of AF was associated with increasing age, a diagnosis of cardiac diseases, signs and symptoms of chronic heart failure, peripheral artery disease and WBC count (Table 3 ).

\section{Complications by AF status}

Overall, during hospitalization, 170 patients (21.0\%) presented at least a complication different from death. AF 
Table 1 Clinical and laboratory characteristics of patients by presence of AF

\begin{tabular}{|c|c|c|c|}
\hline & \multicolumn{2}{|c|}{ Atrial Fibrillation } & \multirow[t]{2}{*}{$p$} \\
\hline & No & Yes & \\
\hline Age (years) & $77 \pm 9$ & $82 \pm 8$ & $<0.001$ \\
\hline Women (\%) & $313(49.5)$ & $85(48.3)$ & 0.799 \\
\hline Weight (Kg) & $71 \pm 14$ & $72 \pm 17$ & 0.440 \\
\hline Height (cm) & $167 \pm 9$ & $166 \pm 8$ & 0.739 \\
\hline BMI $\left(\mathrm{Kg} / \mathrm{m}^{2}\right)$ & $25.6 \pm 4.4$ & $25.5 \pm 5.1$ & 0.943 \\
\hline Living in Nursing Home (\%) & $103(18.1)$ & $33(20.9)$ & 0.489 \\
\hline Functional status (\%) & & & $<0.001$ \\
\hline No functional limitations & $401(66.7)$ & $83(50.9)$ & \\
\hline Dependent-Bedridden & $200(33.3)$ & $80(49.1)$ & \\
\hline $\mathrm{CHA}_{2} \mathrm{DS}_{2}$-VASc (score) & $3.2 \pm 1.5$ & $4.1 \pm 1.5$ & $<0.001$ \\
\hline Anti-arrhythmic drugs (\%) & $8(1.3)$ & $21(11.9)$ & $<0.001$ \\
\hline Anti-platelet agents (\%) & $107(16.9)$ & $20(11.4)$ & 0.079 \\
\hline Beta-blockers (\%) & $149(23.6)$ & $82(46.6)$ & $<0.001$ \\
\hline Digoxin $(\%)$ & $2(0.3)$ & $14(8.0)$ & $<0.001$ \\
\hline Diuretics (\%) & $87(13.8)$ & $65(36.9)$ & $<0.001$ \\
\hline Oral anticoagulants (\%) & $27(4.3)$ & $91(51.7)$ & $<0.001$ \\
\hline VKAs & $11(1.7)$ & $22(12.5)$ & $<0.001$ \\
\hline DOACs & $16(2.5)$ & $69(39.2)$ & $<0.001$ \\
\hline RAS-ant (\%) & 231 (36.6) & $69(39.2)$ & 0.538 \\
\hline Statins $(\%)$ & $146(23.1)$ & 38 (21.6) & 0.686 \\
\hline WHO (\%)—Mild disease & $200(32.3)$ & $50(29.6)$ & \\
\hline Mild disease, $\mathrm{O}_{2}$ support & $238(38.4)$ & $77(45.6)$ & 0.236 \\
\hline Severe / critical disease & $181(29.2)$ & $42(24.9)$ & \\
\hline HR (bpm) & $81 \pm 14$ & $82 \pm 17$ & 0.624 \\
\hline $\mathrm{SAP}(\mathrm{mmHg})$ & $128 \pm 21$ & $127 \pm 13$ & 0.599 \\
\hline DAP (mmHg) & $72 \pm 11$ & $72 \pm 13$ & 0.733 \\
\hline $\mathrm{pH}$ & $7.46 \pm 0.06$ & $7.45 \pm 0.06$ & 0.068 \\
\hline $\mathrm{PaO}_{2}(\mathrm{mmHg})$ & $73 \pm 32$ & $80 \pm 38$ & 0.037 \\
\hline $\mathrm{PaCO}_{2}(\mathrm{mmHg})$ & $36 \pm 11$ & $39 \pm 12$ & 0.042 \\
\hline $\mathrm{HCO}_{3}{ }^{-}(\mathrm{mEq} / \mathrm{L})$ & $25.1 \pm 4.0$ & $25.8 \pm 4.1$ & 0.189 \\
\hline $\mathrm{FiO}_{2}(\%)$ & $34.1 \pm 22.7$ & $36.9 \pm 24.6$ & 0.056 \\
\hline $\mathrm{PaO}_{2} / \mathrm{FiO}_{2}$ & $256 \pm 106$ & $264 \pm 127$ & 0.514 \\
\hline $\mathrm{Hb}(\mathrm{g} / \mathrm{dL})$ & $12.7 \pm 1.9$ & $12.0 \pm 2.0$ & 0.001 \\
\hline $\operatorname{PLT}\left(\mathrm{n} .10^{9} / \mathrm{L}\right)$ & $219 \pm 94$ & $215 \pm 92$ & 0.645 \\
\hline WBC $\left(\mathrm{n} .10^{9} / \mathrm{L}\right)$ & $7.2 \pm 3.5$ & $8.0 \pm 4.9$ & 0.045 \\
\hline Lymphocytes (\%) & $15.8 \pm 10.3$ & $14.9 \pm 9.9$ & 0.351 \\
\hline LDH (U/L) & $373 \pm 207$ & $371 \pm 232$ & 0.933 \\
\hline CRP (mg/L) & $99 \pm 158$ & $89 \pm 162$ & 0.120 \\
\hline INR & $1.2 \pm 0.7$ & $2.0 \pm 2.8$ & $<0.001$ \\
\hline
\end{tabular}

$B M I$ body mass index, $C R P$ C-reactive protein, Dependent-Bedridden severe dependency, $H b$ hemoglobin, $H R$ heart rate, INR international normalized ratio, Mild disease, $\mathrm{O}_{2}$ support mild disease with lowflow oxygen support needed, No functional limitations the patient can walk independently or with the help of a walking-stick, PLT/WBC platelets/white blood cells count, RAS-ant renin-angiotensin system antagonists, SAP/DAP systolic/diastolic arterial pressure, Severe/critical disease needing high-flow oxygen support, non-invasive or invasive mechanical ventilation, or organ support, $\mathrm{WHO}$ WHO classification of severity of COVID-19

Percentages can vary in accordance with missing values. Median number of patients with valid data for each variable: $797 / 808(98.6 \%$; 25th-75th percentile: $97-100$ ) status at baseline did not differ between those with and without events $(p=0.917)$. Severe superinfections $(17.3 \%)$, respiratory failure $(15.8 \%)$, cardiac disturbances $(12.0 \%)$, delirium $(9.8 \%)$, stroke and embolic events $(8.3 \%)$, gastrointestinal bleeding (5.3\%), deep vein thrombosis (3.8\%) and acute renal failure (2.3\%) were the most frequent first diagnosed complications. During hospitalization, 10 more patients (1.6\%) developed their first episode of AF. When compared to the remaining population, they were characterized by older age ( $84 \pm 7$ vs. $77 \pm 9$ years; $p=0.032$ ).

\section{Mortality by AF status}

After excluding two patients with incomplete data, we found that in-hospital mortality was higher in AF than in non-AF subjects ( 36.9 vs. $27.5 \%$; OR $=1.55,95 \% \mathrm{CI}$ $1.09-2.20 ; p=0.015)$.

Length of stay did not differ by rhythm (non-AF $22 \pm 20$ vs. AF $22 \pm 24$ days; $p=0.717$ ), but it was on average lower ( -9 days; 95\% CI 4-14) in those who died (Survived-yes: $24 \pm 22$ vs. No: $15 \pm 14$ days; $p<0.001$; $\mathrm{p}$ for the interaction term $=0.901$ ).

After the exclusion of patients with incomplete data for mobility $(N=44,5.4 \%)$ and/or COVID-19 clinical status at baseline $(N=20,2.5 \%)$, we built the exploratory decision tree analysis model (overall predictivity: $75.1 \%$ ). For AF patients, mobility at baseline was the main factor associated with in-hospital mortality. In particular, $22.8 \%$ and $51.9 \%$ of independent and functionally dependent cases, respectively, died $(p<0.001)$ (Fig. 2). Interestingly, OAC therapy was associated with reduced mortality in subjects with (OAC-Yes: 38.9 vs. No: $63.4 \% ; p=0.041)$ and without (OAC-Yes: 11.4 vs. 37.1\%; $p=0.014)$ disability.

When taking into consideration patients without AF, the first most important factor associated with prognosis was COVID-19 severity at baseline, and secondly, age, sex, and mobility level (Fig. 2). In particular, in subjects without the arrhythmia and with a mild clinical profilenot requiring oxygen at hospital admission-mortality was higher in men than in women. In those receiving oxygen with nasal prongs or face mask, the presence of functional disability was associated with a lower survival rate. Last, non-AF patients with severe-critical conditions at their admission in hospital and older than 78 years showed the highest mortality rate of the entire in-hospital GeroCovid registry $(72.5 \%)$. Interestingly, the group which ranked second in terms of mortality was composed by functionally dependent AF patients. The AUC obtained clustering our study population in accordance with the results of the decision tree analyses was $0.733 \pm 0.020$ (95\% CI $0.693-0.773)$, significantly different from the diagonal reference line $(p<0.001)$. 
Fig. 1 Prevalence of comorbid conditions by AF status in the GeroCovid population. $\mathrm{CHF}$ signs and symptoms of chronic heart failure, $C K D$ chronic kidney disease, $C O P D$ chronic obstructive pulmonary disease, dis. disease, $P A D$ peripheral artery disease

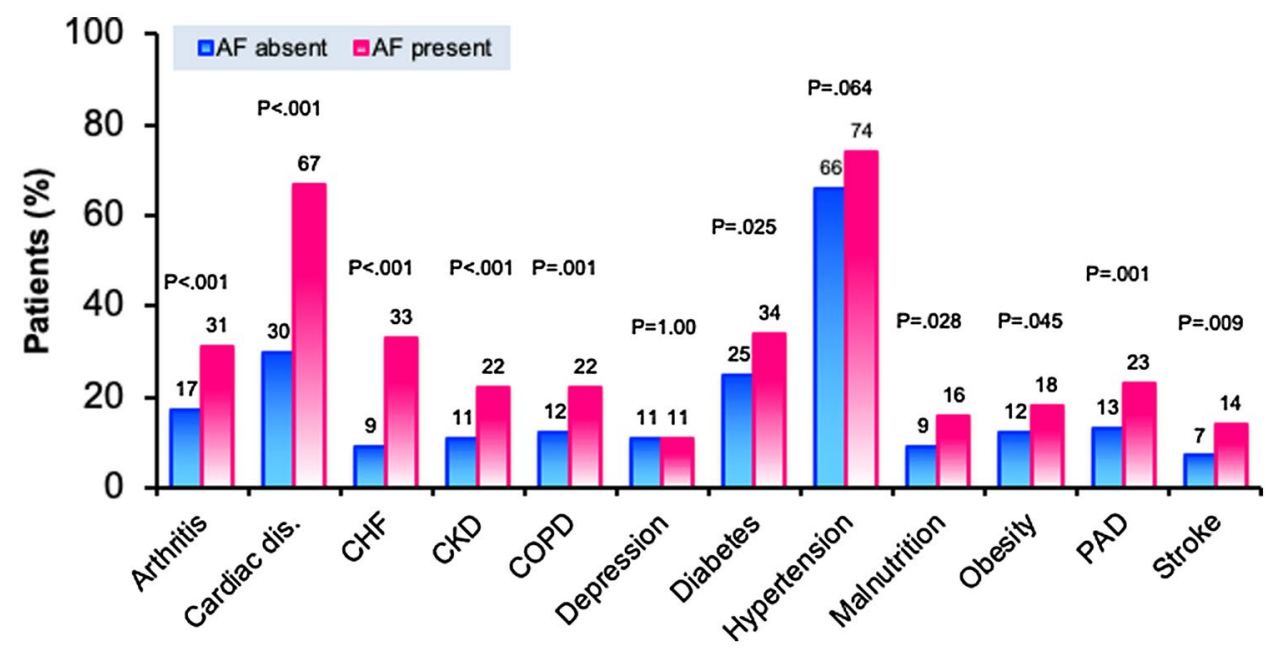

Table 2 Signs and symptoms of COVID-19 at baseline, by presence of AF

\begin{tabular}{llll}
\hline & \multicolumn{2}{l}{ Atrial Fibrillation } & \multirow{2}{*}{$p$} \\
\cline { 2 - 3 } & No & \\
\cline { 2 - 3 } Anorexia (\%) & $68(14.3)$ & $18(13.7)$ & 0.889 \\
Cough (\%) & $291(52.1)$ & $62(40.5)$ & 0.014 \\
Delirium (\%) & $47(9.3)$ & $17(12.6)$ & 0.259 \\
Diarrhea (\%) & $63(12.6)$ & $15(11.2)$ & 0.768 \\
Dyspnea (\%) & $375(65.4)$ & $94(59.1)$ & 0.161 \\
Falls / Fainting (\%) & $19(3.0)$ & $9(1.4)$ & 0.154 \\
Fever (\%) & $388(66.6)$ & $85(53.5)$ & 0.003 \\
Increased respiratory rate (\%) & $266(51.6)$ & $64(44.8)$ & 0.157 \\
Loss of smell (\%) & $6(1.4)$ & $1(0.8)$ & 1.000 \\
Loss of taste (\%) & $10(2.4)$ & $1(0.8)$ & 0.470 \\
Hb O Sat at rest <90\% (\%) & $349(61.8)$ & $92(58.2)$ & 0.461 \\
Hb $\mathrm{O}_{2}$ Sat when walking <90\% (\%) & $258(51.7)$ & $57(41.9)$ & 0.043 \\
Muscles aching (\%) & $41(8.5)$ & $8(6.3)$ & 0.469 \\
Nausea / Vomiting (\%) & $43(8.7)$ & $7(5.3)$ & 0.276 \\
Weakness (\%) & $296(57.1)$ & $74(54.8)$ & 0.697 \\
\hline
\end{tabular}

Percentages can vary in accordance with missing values. Median number of patients with valid data for signs/symptoms of disease at baseline: $634 / 808$ (78.6\%; 25 th-75th percentile: $75.5-83.2)$

$\mathrm{Hb}$ hemoglobin, $\mathrm{O}_{2} \mathrm{Sat} \mathrm{O}_{2}$ saturation

\section{Discussion}

Present findings from the GeroCovid Registry show that among older patients hospitalized for COVID-19, those with $\mathrm{AF}$ at baseline amounted to over $20 \%$ and were older, with a worse functional profile and a higher prevalence of both cardiovascular and non-cardiovascular comorbidities. They also had higher WBC count, but less frequently typical COVID-19 signs and symptoms like cough, fever and desaturation during walking. The proportion of subjects presenting at least one complication different from death during hospitalization was similar in those with and without the arrhythmia. COVID19 severity at presentation did not differ by AF status, but it qualified as a prognostic factor only in non-AF patients. A worse functional profile was associated with reduced survival in AF subjects. Overall, the risk of in-hospital mortality was $55 \%$ higher in $\mathrm{AF}$ than in non-AF patients.

Considering the features of AF patients in our population, we observed that for each one-year increase of age, the probability of having a diagnosis of AF was higher of $4 \%$. Similarly, the arrhythmia was associated with a worse clinical profile and especially a greater prevalence of diabetes and cardiovascular diseases. These results can justify the higher mortality we observed in AF population in GeroCovid, and are strengthened by those of previous works. In the Olmsted County Study, even if in a population younger than ours, the prevalence of all the most important comorbidities was higher in the AF than in the non-AF population [12]. Clinical complexity is one of the reasons advocating the presence of an arrhythmia team for the more appropriate management of older individuals with AF [13]. In keeping with these findings, the higher prevalence of cardiovascular and non-cardiovascular conditions can also explain the greater $\mathrm{CHA}_{2} \mathrm{DS}_{2}$-VASc score and the poorer mobility observed in our AF population. Indeed, in older patients with persistent $\mathrm{AF}$, the $\mathrm{CHA}_{2} \mathrm{DS}_{2}$-VASc score had been correlated with a reduced neuro-cognitive and physical function, and with a higher burden of depressive symptoms [14]. Interestingly, selected comorbidities of AF, like cognitive impairment, anemia, diabetes mellitus and vascular disease qualified as risk factors for COVID-19 infection and its worse prognosis [4].

An interesting result from our experience concerns the WBC count, which was slightly, but significantly, higher in AF subjects. Evidence supporting this finding derives from the Framingham Heart Study and the Atherosclerosis Risk 
Table 3 Variables associated with the presence of AF in the older patients with COVID19 enrolled in the GeroCovid Registry at the multivariable logistic regression analysis (Overall goodness of fit: $82.9 \%$; $p<0.001)$

\begin{tabular}{lrrrr}
\hline & \multicolumn{1}{l}{$\beta \pm$ es } & \multicolumn{1}{c}{ OR } & \multicolumn{1}{c}{$95 \%$ CI } \\
\hline Age $(\Delta$. year) & $0.04 \pm 0.01$ & 0.010 & 1.04 & $1.01-1.07$ \\
Cardiac diseases (Yes vs. No) & $1.05 \pm 0.26$ & $<0.001$ & 2.87 & $1.72-4.79$ \\
Chronic Heart Failure (Yes vs. No) & $0.95 \pm 0.33$ & 0.003 & 2.60 & $1.37-4.93$ \\
Peripheral Artery Disease (Yes vs. No) & $0.66 \pm 0.31$ & 0.030 & 1.94 & $1.06-3.54$ \\
WBC count $\left(\Delta .1 .10^{9} / \mathrm{L}\right)$ & $0.06 \pm 0.03$ & 0.031 & 1.06 & $1.01-1.12$ \\
Constant & $-5.55 \pm 1.12$ & $<0.001$ & 0.004 & - \\
\hline
\end{tabular}

Variables backward deleted from the model-Arthritis: $p=0.256$; CKD: $p=0.279$; COPD: $p=0.164$; Diabetes: $p=0.727$; Functional status: $p=0.234$; Hemoglobin: $p=0.404$; Malnutrition: $p=0.364$; Obesity: $p=0.366$; Stroke: $p=0.617$

95\% CI 95\% Confidence Intervals, Chronic Heart Failure presence of signs and symptoms of chronic heart failure, $O R$ Odds Ratio, $W B C$ white blood cells

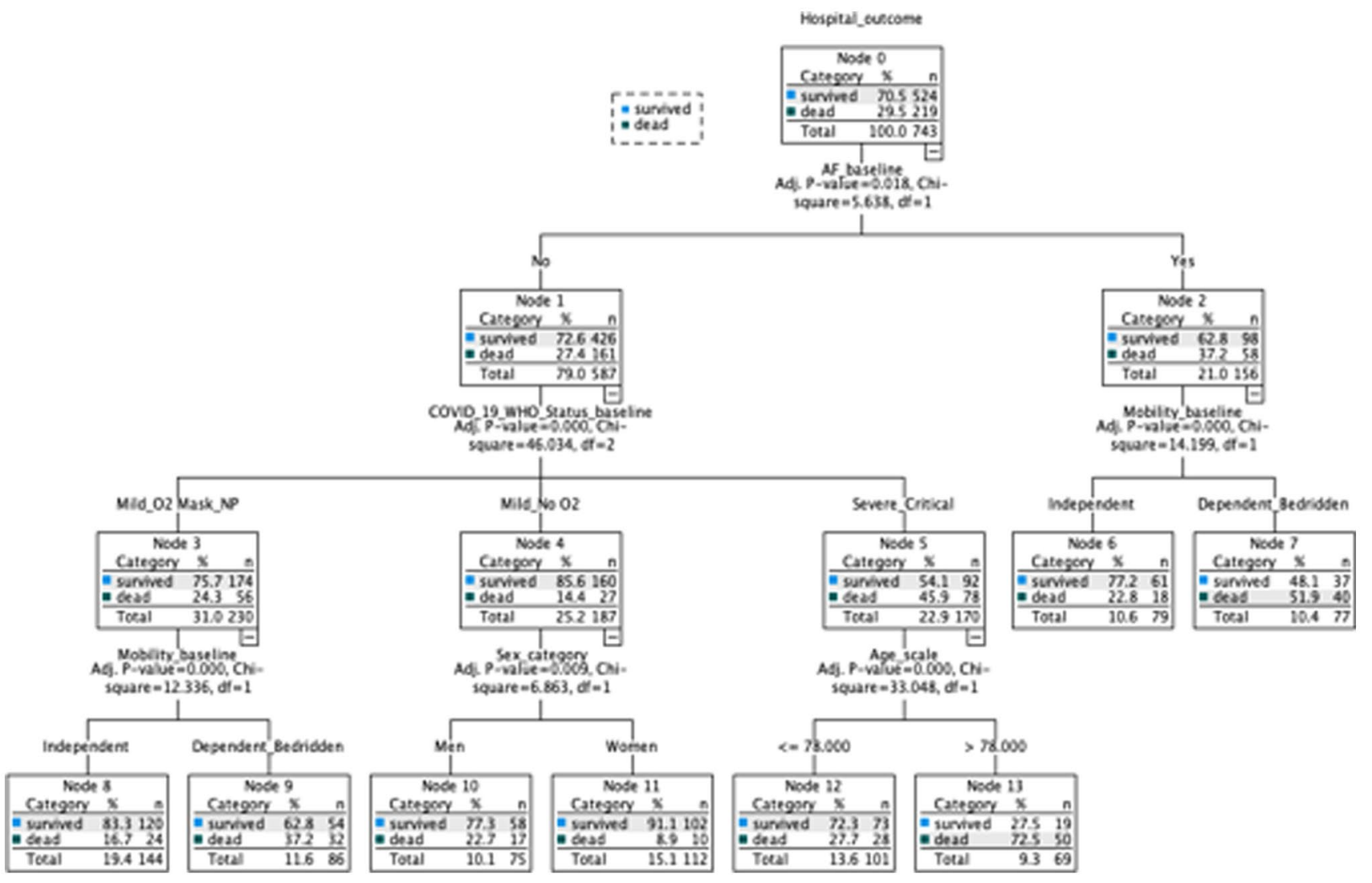

Fig. 2 Tree-based classification model for in-hospital mortality in the GeroCovid population assuming $\mathrm{AF}$ at baseline as the first clustering node. $A F$ atrial fibrillation, Age_scale age, COVID_19_WHO_Status_baseline WHO classification of severity of COVID-19, Mild_No O2 mild disease with no oxygen support needed, Mild_O2 Mask_NP

in Communities (ARIC) study, where leucocytes number correlated with AF onset $[15,16]$.

Some hypothesis can further explain the increased mortality of the older AF patients in GeroCovid. The existence of a connection between inflammation and AF is now well recognized. Furthermore, inflammation itself might qualify as a link between AF and COVID-19. At this regard, left mild disease with low-flow oxygen support (oxygen mask or nasal prongs) needed, Severe_Critical disease needing high-flow oxygen support, non-invasive or invasive mechanical ventilation, or organ support

atrial function was recently found to be lower in hospitalized COVID-19 patients than in COVID-19-negative controls showing a similar degree of respiratory critical illness, with atrial dysfunction closely associated with inflammation [17]. Indeed, the expression of angiotensin-converting enzyme 2 (ACE2) receptors, to which the SARS-CoV-2 binds to enter the cell, is increased in cardiovascular conditions like AF. 
Importantly, many risk factors of AF, such as older age, male gender, obesity, hypertension, diabetes, vascular diseases, left ventricular mass, diastolic dysfunction and atrial disease correlate with ACE2 levels [18] and promote also a low-grade inflammatory status. The interaction of the receptors with the SARS-CoV-2, enhancing the host immune response, was shown to promote myocardial injury and the activation of a procoagulant state, and, ultimately, to reduce survival [19]. These mechanisms, also, might variably contribute to explain the increased mortality observed in our AF subjects ( $36.9 \%$ vs. $27.5 \%$ in non-AF patients). Consistently, in an Italian hospitalized COVID-19 population, mortality was significantly higher (36 vs. $15 \%$ ) in patients with $\mathrm{AF}$ and/or heart failure [20].

In GeroCovid, no differences in COVID-19 disease severity at admission were observed by arrhythmia status. However, as mentioned above, AF patients presented a higher degree of functional dependency, with this factor being significantly associated with mortality and further strengthened by the lack of use of oral anticoagulants [10]. Indeed, some population studies found that the years lived with disability and the disability-adjusted life years are progressively increasing when $\mathrm{AF}$ is present, with a trend closely mirroring the prevalence of the arrhythmia [21]. The greater clinical complexity of patients, the AF-related severe complications and possible changes in cognitive function and depressive symptoms can justify this association [22]. Furthermore, in older persons, the loss of physical functioning was shown to be more closely correlated to mortality than multimorbidity itself [23]. Given that AF subjects showed less frequently cough, fever and exercise hemoglobin desaturation at baseline, an atypical presentation could be the clinical hallmark of COVID-19 patients with AF delaying the care.

Instead, in non-AF patients, WHO classification of clinical status at admission remained the most important factor associated with in-hospital survival. In these individuals, age was associated with mortality in those with more severe COVID-19 clinical presentation, while gender and reduced physical functioning significantly influenced the prognosis of patients with lower disease severity. In line with our results, a previous study found that disability was addictive to age $\geq 80$ years in characterizing prognosis of older seniors hospitalized for SARS-CoV-2 infection. In particular, the group with older age and functional dependency showed a mortality risk 10 times higher than their counterparts [24]. In another experience involving $\geq 75$ years patients, pre-infection disability and frailty significantly improved the accuracy of prognostic models, when added to clinical variables commonly used for younger individuals [25]. Further, in accordance with the existing literature [26], we found that women not needing oxygen support had the lowest mortality rate.

\section{Study limitations and strengths}

We were not able to discriminate among the different types of AF. However, given the advanced age of our population, we can think that persistent or permanent forms of the arrhythmia were prevalent over paroxysmal ones. Because of study design, it was also impossible to differentiate between known and, newly diagnosed, first episodes of $\mathrm{AF}$ at baseline, and to evaluate the use of anticoagulation in these two different types of the arrhythmia. In order to ease the work of health personnel in a very critical environmental context, the collection of not routinely used blood tests was not mandatory. Accordingly, we could not estimate the related differences by AF status. Furthermore, the doses of drugs and oral anticoagulants were not available. For clinical and logistic problems, it was impossible to perform a complete Geriatric Multidimensional Assessment. However, the proportion of subjects living in nursing homes, characterized by a more complex clinical condition and a frequently altered neuro-cognitive function, did not differ by AF status. Lastly, the protective effect of oral anticoagulation might to some extent reflect a selection bias, due to the preferential use of therapy in the fittest population. Nevertheless, we only found a not significantly higher drug prescription rate in the autonomous patients who were enrolled (56.6 vs. $46.3 \% ; p=0.211$ ). On the other hand, the large sample size and the wide set of collected variables represent the strengths of our work, as well as the multicenter nature of the GeroCovid Registry.

In conclusion, our study shows that $\mathrm{AF}$ is a frequent comorbidity in older patients with COVID-19 and it is often associated with complex clinical conditions. Different factors may influence the prognosis of COVID-19 patients with and without AF. Indeed, even if the severity of SARS-CoV-2 infection in $\mathrm{AF}$ and non-AF patients was comparable at ward admission, AF associated with higher in-hospital mortality, which is to some extent related to pre-existing disability. In patients without $\mathrm{AF}$, in-hospital prognosis was mainly related to the severity of infection at admission and, then, to age, sex and functional status. If prospectively confirmed, the stratification approach we obtained could help to evaluate the risk and to guide the most appropriate management of the frailest segment of COVID-19 population.

Acknowledgements We thank Mrs. Gilda Borselli of the Italian Society of Gerontology and Geriatrics (SIGG) for her constant, passionate, help to the activity of the GeroCovid Registry Investigators.

Funding The authors did not receive support from any organization for the submitted work.

Data availability The datasets generated during and/or analysed during the current study are not publicly available due to the privacy of individuals that were enrolled, the particular severe clinical conditions we 
observed in many cases, and the on-going follow-up, but are available from the corresponding author on reasonable request.

\section{Declarations}

Conflict of interest The authors have no conflicts of interest to declare that are relevant to the content of this article.

Statement of human and animal rights The study was approved by the ethics committee of each participating centre, and it was performed in accordance with the ethical standards as laid down in the 1964 Declaration of Helsinki and its later amendments or comparable ethical standards.

Informed consent Informed consent was obtained from all participants included in the study or from their legal caregivers.

\section{References}

1. Magnussen C, Niiranen TJ, Ojeda FM et al (2017) Sex Differences and similarities in atrial fibrillation epidemiology, risk factors, and mortality in community cohorts: results from the BiomarCaRE consortium (biomarker for cardiovascular risk assessment in Europe). Circulation 136:1588-1597. https://doi.org/10.1161/CIRCULATIO NAHA.117.028981

2. Fumagalli C, Rozzini R, Vannini M et al (2020) Clinical risk score to predict in-hospital mortality in COVID-19 patients: a retrospective cohort study. BMJ Open 10:e040729. https://doi.org/10.1136/bmjop en-2020-040729

3. O'Shea CJ, Middeldorp ME, Thomas G et al (2021) Atrial fibrillation burden during the coronavirus disease 2019 pandemic. Europace. https://doi.org/10.1093/europace/euab099

4. Lip GYH, Genaidy A, Tran G et al (2021) Incident atrial fibrillation and its risk prediction in patients developing COVID-19: a machine learning based algorithm approach. Eur J Intern Med. https://doi.org/ 10.1016/j.ejim.2021.04.023

5. García-Granja PE, Veras C, Aparisi Á et al (2021) Atrial fibrillation in patients with SARS-CoV-2 infection. Med Clin (Engl Ed) 157:58-63. https://doi.org/10.1016/j.medcle.2021.01.010

6. Zuin M, Rigatelli G, Bilato C et al (2021) Pre-existing atrial fibrillation is associated with increased mortality in COVID-19 patients. J Interv Card Electrophysiol 62:231-238. https://doi.org/10.1007/ s10840-021-00992-2

7. Peltzer B, Manocha KK, Ying X et al (2020) Outcomes and mortality associated with atrial arrhythmias among patients hospitalized with COVID-19. J Cardiovasc Electrophysiol 31:3077-3085. https:// doi.org/10.1111/jce.14770

8. Paris S, Inciardi RM, Lombardi CM et al (2021) Implications of atrial fibrillation on the clinical course and outcomes of hospitalized COVID-19 patients: results of the Cardio-COVID-Italy multicentre study. Europace 23:1603-1611. https://doi.org/10.1093/europace/ euab146

9. Spinoni EG, Mennuni M, Rognoni A et al (2021) Contribution of atrial fibrillation to in-hospital mortality in patients with COVID-19. Circ Arrhythm Electrophysiol 14:e009375. https://doi.org/10.1161/ circep. 120.009375

10. Fumagalli S, Trevisan C, Del Signore S et al (2021) COVID-19 and atrial fibrillation in older patients: does oral anticoagulant therapy provide a survival benefit?-An insight from the GeroCovid registry. Thromb Haemost. https://doi.org/10.1055/a-1503-3875

11. Trevisan C, Del Signore S, Fumagalli S et al (2021) Assessing the impact of COVID-19 on the health of geriatric patients: the European GeroCovid observational study. Eur J Intern Med 87:29-35. https://doi.org/10.1016/j.ejim.2021.01.017
12. Chamberlain AM, Alonso A, Gersh BJ et al (2017) Multimorbidity and the risk of hospitalization and death in atrial fibrillation: a population-based study. Am Heart J 185:74-84. https://doi.org/10. 1016/j.ahj.2016.11.008

13. Fumagalli S, Chen J, Dobreanu D et al (2016) The role of the arrhythmia team, an integrated, multidisciplinary approach to treatment of patients with cardiac arrhythmias: results of the European heart rhythm association survey. Europace 18:623-627. https://doi. org/10.1093/europace/euw090

14. Fumagalli S, Pelagalli G, Montorzi RF et al (2020) The CHA2DS2VASc score and geriatric multidimensional assessment tools in elderly patients with persistent atrial fibrillation undergoing electrical cardioversion. A link with arrhythmia relapse? Eur J Intern Med 82:56-61. https://doi.org/10.1016/j.ejim.2020.07.010

15. Rienstra M, Sun JX, Magnani JW et al (2012) White blood cell count and risk of incident atrial fibrillation (from the Framingham Heart Study). Am J Cardiol 109:533-537. https://doi.org/10.1016/j.amjca rd.2011.09.049

16. Misialek JR, Bekwelem W, Chen LY et al (2015) Association of white blood cell count and differential with the incidence of atrial fibrillation: the atherosclerosis risk in communities (ARIC) study. PLoS ONE 10:e0136219. https://doi.org/10.1371/journal.pone. 0136219

17. Goerlich E, Minhas A, Gilotra N et al (2021) Left atrial function in patients with COVID-19 and its association with incident atrial fibrillation/flutter. J Am Soc Echocardiogr. https://doi.org/10.1016/j. echo.2021.05.015

18. Sanchis-Gomar F, Perez-Quilis C, Lavie CJ (2020) Should atrial fibrillation be considered a cardiovascular risk factor for a worse prognosis in COVID-19 patients? Eur Heart J 41:3092-3093. https:// doi.org/10.1093/eurheartj/ehaa509

19. Inciardi RM, Adamo M, Lupi L et al (2020) Atrial fibrillation in the COVID-19 era: simple bystander or marker of increased risk? Eur Heart J 41:3094. https://doi.org/10.1093/eurheartj/ehaa576

20. Inciardi RM, Adamo M, Lupi L et al (2020) Characteristics and outcomes of patients hospitalized for COVID-19 and cardiac disease in Northern Italy. Eur Heart J 41:1821-1829. https://doi.org/10.1093/ eurheartj/ehaa388

21. Roth GA, Mensah GA, Johnson CO et al (2020) Global burden of cardiovascular diseases and risk factors, 1990-2019: update from the GBD 2019 study. J Am Coll Cardiol 76:2982-3021. doi:https://doi. org/10.1016/j.jacc.2020.11.010

22. Fumagalli S, Pelagalli G, Franci Montorzi R et al (2021) Atrial fibrillation in older patients and artificial intelligence: a quantitative demonstration of a link with some of the geriatric multidimensional assessment tools-a preliminary report. Aging Clin Exp Res 33:451-455. https://doi.org/10.1007/s40520-020-01723-9

23. Landi F, Liperoti R, Russo A et al (2010) Disability, more than multimorbidity, was predictive of mortality among older persons aged 80 years and older. J Clin Epidemiol 63:752-759. https://doi. org/10.1016/j.jclinepi.2009.09.007

24. Plotnikov G, Waizman E, Tzur I et al (2021) The prognostic role of functional dependency in older inpatients with COVID-19. BMC Geriatr 21:219. https://doi.org/10.1186/s12877-021-02158-1

25. Fumagalli C, Ungar A, Rozzini R et al (2021) Predicting mortality risk in older hospitalized persons With COVID-19: a comparison of the COVID-19 mortality risk score with frailty and disability. J Am Med Dir Assoc 22:1588-1592 e1581. https://doi.org/10.1016/j. jamda.2021.05.028

26. Biolè C, Bianco M, Núñez-Gil IJ et al (2021) Gender differences in the presentation and outcomes of hospitalized patients with COVID19. J Hosp Med 16:349-352. https://doi.org/10.12788/jhm.3594

Publisher's Note Springer Nature remains neutral with regard to jurisdictional claims in published maps and institutional affiliations. 